\title{
Abnormal biomechanics: a precursor or result of knee osteoarthritis?
}

\section{A Teichtahl, A Wluka, F M Cicuttini}

\section{Biomechanical studies are required to differentiate between the causes and results of knee osteoarthritis}

A lthough osteoarthritis (OA) is a common cause of disability in people over 65 years, ${ }^{1}$ the causes and pathogenesis of knee OA remain largely unknown. In addition to biological studies, there is increasing interest in the contribution of biomechanical variables in the pathogenesis and management of this disease. ${ }^{2-6}$

OA of the knee occurs most commonly in the medial tibiofemoral compartment, ${ }^{3}$ and increased regional load across this compartment's articular cartilage is believed to be an important factor in the pathogenesis of the disease. ${ }^{2-6}$ The external knee adduction moment is argued to distribute $60-80 \%$ of total intrinsic knee compressive load to the medial tibiofemoral compartment, ${ }^{3}$ and people with medial tibiofemoral OA tend to walk with larger knee adduction moments than normal subjects, resulting in increased medial compartment pressure. $^{27}$ Despite this, there is no clear evidence to suggest whether biomechanical abnormalities such as increased knee adduction moments cause or occur as a result of OA because all previous studies have examined people with established disease.

The knee adduction moment is generated by the combination of the ground reaction force, which passes medial to the centre of the knee joint, and the perpendicular distance of this force from the centre of the joint.' Given that varus alignment of the lower limb theoretically increases the perpendicular distance of the ground reaction force from the centre of the knee joint, it is not surprising that radiographic varus alignment is associated with the magnitude of the peak knee adduction moment in subjects with healthy and osteoarthritic knees. ${ }^{568}$ However, only 50\% of knee adduction moment variability in subjects with medial tibiofemoral OA is accounted for by the mechanical axis of the lower limb, emphasising the need for dynamic evaluation of the knee joint loading environment. ${ }^{5}$ Preliminary studies have shown a moderately strong and significant association between both the line of progression and degree of foot rotation during gait and the magnitude of the knee adduction moment. ${ }^{58}$ Further examination of dynamic factors associated with the knee adduction moment is required to help better understand the biomechanical pathogenesis of knee OA.

Although biomechanical factors are likely to contribute to the causes and pathogenesis of knee OA, their effect on joint morphology is unknown. Whereas increased mechanical load at the knee results in increased bone mineral density, ${ }^{10}$ little is understood about cartilage response to repetitive altered load. Previous studies on people with knee OA showed that a larger knee adduction moment was associated with greater medial joint space narrowing. ${ }^{26} \mathrm{How}$ ever, because knee joint space consists of other structures such as menisci, joint space narrowing is not always a valid indicator of articular cartilage volume. ${ }^{11}$ Although there is emerging evidence that cartilage volume will be a useful measure in studies of the pathogenesis of $\mathrm{OA}^{12-15}$ future work must examine how human tissues, including hyaline cartilage, respond to altered biomechanical variables such as the knee joint loads experienced during locomotion.

\section{"Quadriceps weakness has long been anecdotally recognised as a feature common to knee OA."}

While joint compressive forces such as the knee adduction moment have received considerable attention in recent times, the relation between muscle weakness and knee OA is also becoming better understood. Quadriceps weakness has long been anecdotally recognised as a feature common to knee OA. Although longitudinal studies have shown that quadriceps weakness is a characteristic of people with established knee OA, weakness is also likely to be a risk factor for the development of disease. ${ }^{16}{ }^{17}$ A previous study showed that baseline knee extensor strength was lower in women without radiographic knee OA at initial examination who later developed OA changes, compared with unaffected strength during gait reduces the net women. ${ }^{18}$ It may be that weak quadriceps extensor moment, which may help to counteract the lateral knee joint opening and medial compression that would occur if the knee adduction moment acted as an unopposed force. Although it may be useful to determine whether there is an association between quadriceps strength and the knee adduction moment during gait, further longitudinal studies are required to substantiate whether quadriceps weakness is a major cause or effect of knee OA.

Knee joint laxity, which is defined as displacement or rotation of the tibia with respect to the femur, ${ }^{18}$ is another biomechanical variable argued to contribute to the pathogenesis of OA. One study showed that varus-valgus laxity is greater in the unaffected knees of patients with unilateral OA than in healthy control subjects, ${ }^{19}$ suggesting that knee joint laxity may predispose to disease. However, such presumptions rely on the subjects with unilateral knee OA developing bilateral disease, which may not transpire. Nonetheless, it has been shown that varus and valgus alignment of the lower limb is associated with the progression of medial and lateral compartment knee OA, as determined by joint space narrowing and deterioration of physical function. ${ }^{20}$ Moreover, changes resulting from the relation between alignment and disease progression can be detected after only 18 months of observation..$^{20}$ This suggests that over a relatively short time frame of intervention, the correction of biomechanical variables in people with established knee OA may delay the progression of disease.

Recently, a study combined several of the previously discussed variables and examined the role of quadriceps strength in the progression of knee OA using subjects with malaligned and lax knee joints. $^{21}$ Although earlier results suggested that women with reduced quadriceps strength have a greater risk of developing knee OA, ${ }^{16}$ Sharma et $a^{21}$ concluded that greater quadriceps strength at baseline was associated with increased likelihood of OA progression in malaligned and lax knees. Although these results infer that strong quadriceps reduce the risk of developing knee OA, they also suggest that strong quadriceps are a risk factor for the progression of disease in people with malaligned and lax arthritic knees.

Biomechanical factors are increasingly being recognised as potential contributors to the causes and pathogenesis of knee OA. Until recently most studies on the biomechanics of knee OA have tended to be cross sectional rather than longitudinal, making it difficult to differentiate between the factors that are a cause or result of the disease. Longitudinal studies in normal subjects are required to determine whether biomechanical variables, such as the knee 
adduction moment, predate the onset of OA or occur after disease is present. Other studies in subjects with OA will be required to clarify the role of biomechanical variables in disease progression, to identify potentially modifiable factors to alter the course of disease. Once these data are available, simple interventions such as gait re-education or orthoses may provide a future strategy for modifying the biomechanical risk factors associated with the onset or progression of knee OA.

Br J Sports Med 2003;37:289-290

\section{Authors' affiliations}

A Teichtahl, A Wluka, F M Cicuttini, Monash University, Melbourne, Australia

Correspondence to: Associate Professor Cicuttini, 3rd Floor, 553 St Kilda Rd, Melbourne, Vic 3001, Australia;

flavia.cicuttini@med.monash.edu.au

\section{REFERENCES}

1 Felson DT, Naimark A, Anderson J, et al. The prevalence of knee osteoarthritis in the elderly. The Framingham Osteoarthritis Study. Arthritis Rheum 1987:30:914-18.

2 Sharma L, Hurwitz DE, Thonar EJMA, et al Knee adduction moment, serum hyaluronan level, and disease severity in medial tibiofemoral osteoarthritis. Arthritis Rheum 1998:41:1223-40.
3 Andriacchi TP, Dynamics of knee malalignment. Orthop Clin North Am 1994;25:395-403.

4 Kaufman KR, Hughes C, Morrey BF, et al. Gait characteristics of patients with knee osteoarthritis. J Biomech 2001;34:907-15.

5 Hurwitz DE, Ryals AB, Case JP, et al. The knee adduction moment during gait in subjects with knee osteoarthritis is more closely correlated with static alignment than radiographic disease severity, toe out angle and pain. J Orthop Res 2002;20:101-7.

6 Miyazaki T, Wada M, Kawahara $\mathrm{H}$, et al. Dynamic load at baseline can predict radiographic disease progression in medial compartment knee osteoarthritis. Ann Rheum Dis 2002:61:617-22.

7 Schipplein OD, Andriacchi TP. Interaction between active and passive knee stabilizers during level walking. J Orthop Res 1991;9:113-19.

8 Andrews $M$, Noyes FR, Hewett TE, et al. Lower limb alignment and foot angle are related to stance phase knee adduction in normal subjects: a critical analysis of the reliability of gait analysis data. J Orthop Res 1996:14:289-95.

9 Wada M, Maezawa Y, Baba H, et al. Relationships among bone mineral densities static alignment and dynamic load in paients with medial compartment knee osteoarthritis. Rheumatology 2001;40:499-505.

10 Adams JG, McAlindon T, Dimasi M, et al. Contribution of meniscal extrusion and cartilage loss to joint space narrowing in osteoarthritis. Clin Radiol 1999;54:502-6.

11 Peterfy CG, van Dijke CF, Janzen DL, et al. Quantification of articular cartilage in the knee with pulsed saturation transfer subtraction and fat-suppressed MR Imaging: optimization and validation. Radiology 1994; 192:485-91.
12 Eckstein F, Westhoff J, Sittek H, et al. In vivo reproducibility of three-dimensional cartilage volume and thickness measurements with MR imaging. Am J Radiol 1998;170:593-7.

13 Jones G, Glisson M, Hynes K, et al. Sex and site differences in cartilage development: a possible explanation for variations in knee osteoarthritis in later life. Arthritis Rheum 2000;43:2543-9.

14 Raynauld JP, Pelletier JP, Beaudoin G, et al. A two year study in osteoarthritis patients following the progression of the disease by magnetic resonance imaging using a novel quantification imaging system. Arthritis Rheum 2002;46:311

15 Slemenda C, Heilman DK, Brandt KD, et al. Reduced quadriceps strength relative to body weight: a risk factor for knee osteoarthritis in women? Arthritis Rheum 1998;41:1951-9.

16 Brandt KD, Heilman MS, Slemenda C, et al. Quadriceps strength in women with radiographically progressive osteoarthritis of the knee and those with stable radiographic changes. J Rheumatol; 1999;26:2431-7.

17 Felson DT, Lawrence RC, Dieppe PA, et al Osteoarthritis: new insights. Part 1. The disease and its risk factors. Ann Intern Med 2000; 133:635-46.

18 Sharma L, Lou C, Felson DT, et al. Laxity in healthy and osteoarthritic knees. Arthritis Rheum 1999:42:861-70.

19 Sharma L, Song J, Felson DT, et al. The role of knee alignment in disease progression and functional decline in knee osteoarthritis. JAMA 2001;286: 188-95.

20 Sharma L, Dunlop DD, Cahue S, et al. Quadriceps strength and osteoarthritis progression in malaligned and lax knees. Ann Intern Med 2003;138:613-19. 